\title{
NT-proBNP increase during stress echocardiography predicts significant changes in ischemic mitral regurgitation severity in patients qualified for surgical revascularization
}

\author{
Radosław Piątkowski, Janusz Kochanowski, Monika Budnik, \\ Marcin Grabowski, Piotr Ścisło, Grzegorz Opolski \\ $1^{\text {st }}$ Chair and Department of Cardiology, Medical University of Warsaw, Poland
}

\begin{abstract}
Background: In many patients, significant changes in ischemic mitral regurgitation (IMR) severity during exercise can be observed independent of the degree of IMR at rest. This study aimed to investigate the correlations between $N$-terminal fragment $B$-type natriuretic peptide (NT-proBNP) and echocardiography measurements at rest and at peak exercise in patients with moderate IMR who qualified for surgical revascularization.
\end{abstract}

Methods: A total of 100 patients eligible for coronary artery bypass grafting, were included in this prospective study. All patients underwent exercise echocardiography. Additionally, the levels of NT-proBNP were measured at rest and after peak exercise.

Results: A positive correlation of absolute NT-proBNP levels with effective regurgitant orifice area (EROA) were observed and with tricuspid regurgitant peak gradient (TRPG) at peak exercise. Absolute $\triangle N T$-proBNP during exercise and the tenting area at rest were independent predictors of severe IMR at peak exercise. The level of absolute $\triangle N T$-proBNP during exercise and coaptation height at rest were the most important predictors of significant increases in TRPG. The best cutoff value for $\triangle N T$-proBNP as a predictor for increases in EROA at peak exercise was $68.9 \mathrm{pg} / \mathrm{mL}$ and to predict an increase in $T R P G \geq 50 \mathrm{mmHg}$ at peak exercise was $68 \mathrm{pg} / \mathrm{mL}$.

Conclusions: The level of $\triangle N T$-proBNP during exercise was the most important parameter in predicting significant changes in IMR severity and pulmonary pressure. Based on the present data, it can be speculated that integration of the assessment of NT-proBNP at rest and at exercise might improve patient selection for valve surgery. (Cardiol J 2022; 29, 6: 927-935)

Key words: natriuretic peptides, ischemic mitral regurgitation, exercise echocardiography

\section{Introduction}

Secondary ischemic mitral regurgitation (IMR) has a dynamic nature. In many patients, significant changes in IMR severity during exercise can be observed independent of the degree of IMR at rest [1-3]. An exercise-induced increase in IMR severity is related to left ventricle (LV) remodeling and mitral valve deformation indices, namely, tenting area $(\mathrm{TA})$ and coaptation height $(\mathrm{CH})$, as well as LV synchronicity [4-6].

Natriuretic peptides such as B-type natriuretic peptide (BNP) and its N-terminal fragment (NT-proBNP) are released from ventricular and atrial myocardium in response to increased wall stress (left and right ventricle) and left atrial pressures due to increasing severity of mitral regurgitation [7-11]. Previous studies have suggested that BNP

Address for correspondence: Radosław Piątkowski, MD, PhD, $1^{\text {st }}$ Chair and Department of Cardiology, Medical University of Warsaw, ul. Banacha 1a, 02-097 Warszawa, Poland, tel: +48 22599 2958, e-mail: radekp1@gmail.com

Received: 8.03.2019 Accepted: 21.04.2020 Early publication date: 3.06.2020

This article is available in open access under Creative Common Attribution-Non-Commercial-No Derivatives 4.0 International (CC BY-NC-ND 4.0) license, allowing to download articles and share them with others as long as they credit the authors and the publisher, but without permission to change them in any way or use them commercially. 
or NT-proBNP levels increase with exercise in patients with coronary artery disease [12], heart failure [13], organic mitral regurgitation [14], and mitral stenosis [15]. However, NT-proBNP levels during exercise do not provide new information on the severity of aortic stenosis [16]. The value of serial measurements of NT-proBNP at rest and during exercise in patients with IMR has not yet been evaluated.

Optimal management of moderate IMR in patients qualified for coronary artery bypass grafting $(\mathrm{CABG})$ is still controversial [17, 18]. Hypothesized herein, that the absolute increase in NT-proBNP with exercise is a reliable parameter in predicting a significant increase in IMR severity and pulmonary systolic artery pressure and may help in the identification of high-risk patients with moderate IMR qualified for CABG. This would help improve risk stratification and identification of the subgroups of patients who could benefit from various surgical strategies (CABG or CABG with mitral repair).

The main purpose of the present study was to assess the correlation between NT-proBNP levels and echocardiography measurements at rest and at peak exercise in patients with moderate IMR, qualified for surgical revascularization.

\section{Methods}

\section{Study population}

A total of 100 patients (mean age $64.4 \pm 7.9$ years) with a history of myocardial infarction (MI) and were eligible for CABG were included in a prospective study. The time frame for enrollment of patients into the study was 24 months. All patients had moderate IMR caused by restrictive systolic leaflet motion (Carpentier's type IIIb), with or without annular dilatation, which occurred in $\geq 16$ days from MI, with no evidence of primary leaflet, chordal, or papillary muscle pathology, excluding mechanical complications of MI.

The study inclusion criterion was the presence of a significant area of viable myocardium, as seen in the improvement in wall motion of at least four dysfunctional segments during dobutamine stress echocardiography (DSE). Exclusion criteria included left bundle branch block, unstable angina, prosthetic heart valve, other valvular or congenital heart diseases, renal failure (creatinine $>2 \mathrm{mg} / \mathrm{dL}$ ), history of CABG, severe heart failure symptoms (New York Heart Association [NYHA] IV), clinical limitations to exercise testing, and atrial fibrillation before exercise testing.
Echocardiographic and clinical assessment were performed before surgery.

Each patient signed an informed consent form, and the study was approved by the institutional review board of the Medical University of Warsaw. The study had been conducted according to the principles stated in the Declaration of Helsinki.

\section{Clinical data}

Clinical assessment was comprised of a careful exploration of patient history and physical examination and was performed at the time of enrollment. Experienced cardiologists blinded to the results of NT-proBNP measurements and echocardiographic findings assessed the functional status. Clinical status was determined according to the criteria of the NYHA classification system and the Canadian Cardiovascular Society (CCS) functional class for heart failure and angina symptoms, respectively.

\section{Echocardiographic measurements and calculations}

Transthoracic echocardiograms (TTE) were performed within 2 days before surgery. All patients underwent TTE in the left lateral decubitus position at rest and during DSE. For accuracy purposes, the images obtained at rest and during stress tests were independently analyzed with a blinded method by two experienced echocardiographers.

All examinations were carried out using the iE33 system manufactured by Philips, a broadband transducer for TTE of 2.5 to $3.5 \mathrm{MHz}$ frequencies. Echocardiographic measurements were averaged over three cardiac cycles.

Ischemic mitral regurgitation severity was assessed by measuring the effective regurgitant orifice area (EROA), with EROA $>10 \mathrm{~mm}^{2}$ and $<20 \mathrm{~mm}^{2}$ considered moderate and EROA $\geq 20 \mathrm{~mm}^{3}$ considered severe, as well as mitral regurgitation volume (MRvol) with MRvol $\geq 30 \mathrm{~mL}$ considered severe [18-20]. EROA and MRvol was calculated using flow convergence (proximal isovelocity surface area-proximal isovelocity surface area [PISA] method). The radius of the PISA (r) is measured from the vena contracta level to the point of color Doppler aliasing. EROA is calculated as: $6.28 \times \mathrm{r}^{2}$ $\times \mathrm{Va} /$ Peak V RegJet, where Va is aliasing velocity and VRegJet is the peak velocity of the regurgitant jet by Continuous Wave Doppler. The MRvol is calculated as EROA $\times$ VTI RegJet where VTI RegJet is the VTI of the regurgitant jet. Wall motion abnormalities were evaluated in accordance with the recommendations of the American Society 
of Cardiology. The wall motion score index was calculated according to a 17-segment model [21]. The left ventricular volumes and ejection fraction (EF) were assessed by the biapical Simpson disk method [22]. Sphericity indices (SI) were obtained at end-diastole (SId) and end-systole (SIs) in the apical view. Pulmonary systolic arterial pressure can be estimated from the maximal tricuspid regurgitant peak gradient (TRPG) using the simplified Bernoulli equation $\left(\Delta \mathrm{P}=4 \mathrm{~V}^{2}\right.$, where $\mathrm{V}=$ maximal tricuspid regurgitant velocity [TRV] in $\mathrm{m} / \mathrm{s}$ ), adding an assumed right atrial pressure which can be estimated by echocardiography based on the diameter and respiratory variation in diameter of the inferior vena cava. However, TRV $>3.4 \mathrm{~m} / \mathrm{s}$ indicates a high probability of pulmonary hypertension. In the present analysis it was assumed that TRPG $\geq 50 \mathrm{mmHg}$ means significant pulmonary hypertension [23]. Mitral valve deformation was evaluated by measuring TA, i.e., the area enclosed between mitral leaflets and the line of annular plane, and $\mathrm{CH}$, i.e., the distance between leaflet coaptation and mitral annular plane from the parasternal long-axis view at mid-systole [24].

Low-dose DSE was used in IMR patients to distinguish akinetic viable segments from nonviable myocardial regions of the LV [25]. DSE was performed in accordance with current guidelines [26]. A graded dobutamine infusion started at $5 \mu \mathrm{g} / \mathrm{kg} / \mathrm{min}$ and was increased at 3-min intervals to $10,20 \mu \mathrm{g} / \mathrm{kg} / \mathrm{min}$.

\section{Exercise echocardiography}

All subjects underwent a symptom-limited graded exercise echocardiography test to assess the dynamics of IMR changes and TRPG, the latter as the exponent of right ventricle overload. The symptom-limited grade exercise echocardiography was performed according to the following protocol: the initial workload of 25 watts (W) was maintained for $3 \mathrm{~min}$, and then the workload was increased every $2 \mathrm{~min}$ by $25 \mathrm{~W}$. Blood pressure and a 12-lead electrocardiogram was recorded every 2 min. Two-dimensional and Doppler echocardiographic recordings were available throughout the test. Exercise was interrupted when ischemic electrocardiographic signs, fatigue, or intolerable dyspnea appeared [24].

\section{NT-proBNP measurements}

Venous blood samples were collected from an antecubital vein and placed into chilled EDTA tubes before and immediately after exercise. The specimens were centrifuged within half an hour at $-4^{\circ} \mathrm{C}$ and then plasma was frozen at $-80^{\circ} \mathrm{C}$ until analysis. NT-proBNP was measured by an electrochemiluminescence immunoassay (ProBNP Elecsys $^{\circledR}$, Roche Diagnostics GmbH). The absolute increase in NT-proBNP during exercise (absolute $\triangle$ NT-proBNP) was calculated as the difference between NT-proBNP at peak exercise and NT-proBNP at rest. An NT-proBNP value of $125 \mathrm{pg} / \mathrm{mL}$ was used to differentiate between normal and abnormal NT-proBNP levels.

\section{Statistical analysis}

All data were prospectively recorded using the institution's database. Continuous variables are presented as mean \pm standard deviation (SD), and categorical variables are presented as either absolute numbers or percentages. The Spearman correlation coefficient was used to assess the relation between NT-proBNP changes and exercise echo parameters. Multiple linear regression analysis was used to identify important predictors of significant increase of IMR severity (EROA exe $\geq 20 \mathrm{~mm}^{2}$ ) and probability of pulmonary hypertension (TRPG $\geq 50 \mathrm{mmHg}$ ) at peak exercise echocardiography examination. Stepwise logistic regression analysis included age, NT-proBNP at rest and at peak exercise, and $\triangle$ NT-proBNP, as well as some echocardiography parameters at rest and at peak exercise, namely, EROA, MRvol, TRPG, TA, $\mathrm{CH}$, left ventricular end-systolic volume (ESV), $\mathrm{EF}$, and SIs. A receiver-operating characteristic (ROC) curve analysis was performed to identify the optimal cutoff point of NT-proBNP levels (at which sensitivity and specificity were maximal) to predict the increase in IMR severity and pulmonary systolic arterial pressure at peak exercise. Optimal cutoff values were determined as the rounding cutoff that gives the maximum sum of sensitivity and specificity. This value should be the shoulder at the top left of the ROC curve. The area under the curve (AUC) value was calculated as a measure of accuracy of the test. ROC analysis was performed for NT-proBNP at rest, NT-proBNP at peak exercise (NT-proBNP exe), and absolute $\triangle$ NT-proBNP as predictors of reaching the above-mentioned echo endpoints (EROA exe $\geq 20 \mathrm{~mm}^{2}$ and TRPG $\geq 50$ $\mathrm{mmHg}$ at peak exercise). The AUCs of the ROC curve for NT-proBNP at rest, NT-proBNP exe, and $\triangle \mathrm{NT}$-proBNP are given together with their $95 \%$ confidence intervals $(95 \% \mathrm{CI})$ and are compared by the nonparametric test proposed by DeLong et al. [27]. A p value of $<0.05$ was considered significant. Statistical analyses were performed with SAS software version 8.02 (SAS Institute Inc., Cary, NC). 
Table 1. Baseline clinical characteristics - all groups $(n=100)$.

\begin{tabular}{lc}
\hline Age [years] & $64.4 \pm 7.9$ \\
BMI $\left[\mathrm{kg} / \mathrm{m}^{2}\right]$ & $27.1 \pm 4.0$ \\
Sex, male & $56(56 \%)$ \\
NT-proBNP at rest, & $769.5(395.5-1334)$ \\
median $[\mathrm{pg} / \mathrm{mL}]$ & \\
NT-proBNP exe, & $789.9(429.9-1478)$ \\
median [pg/mL] & \\
NNT-proBNP, & $30.1(14.7-89)$ \\
median [pg/mL] & \\
NYHA: & $2.0 \pm 0.8$ \\
I & $28(28 \%)$ \\
II & $47(47 \%)$ \\
III & $25(25 \%)$ \\
EuroSCORE Logistic $[\%]$ & $7.1 \pm 5.1$ \\
Smoking (actual) & $15(15 \%)$ \\
Hypertension & $67(67 \%)$ \\
Diabetes mellitus & $32(32 \%)$ \\
Hyperlipidemia & $67(67 \%)$ \\
Atrial fibrillation & $17(17 \%)$ \\
Two-vessel disease & $27(27 \%)$ \\
Three-vessel disease & $70(70 \%)$ \\
ACEl & $94(94 \%)$ \\
Beta-adrenolytics & $96(96 \%)$ \\
Statins & $96(96 \%)$ \\
Acetylsalicylic acid & $91(91 \%)$ \\
\hline
\end{tabular}

Data are presented as mean \pm standard deviation or number (percentage) as shown; ACEI - angiotensin converting enzyme inhibitors; $\mathrm{BMI}$ - body mass index; exe — at peak exercise; NT-proBNP

- N-terminal fragment B-type natriuretic peptide; $\Delta$ NT-proBNP

- difference between NT-proBNP level at peak exercise and

NT-proBNP level at rest; NYHA - New York Heart Association

\section{Results}

The mean age of the patients was $64.4 \pm$ \pm 7.9 years. Of the 100 patients analyzed, 56 were men and 44 were women. The mean logistic EuroSCORE was $7.1 \pm 5.1 \%$. All the patients were given optimal pharmacological treatment. Table 1 shows the clinical characteristics and NT-proBNP levels, and Table 2 the echocardiographic variables at rest and at peak exercise of the IMR patients. Table 3 presents correlation coefficients for associations between NT-proBNP (rest, exercise, and delta) and echocardiographic measures at rest and at peak exercise. Significant negative correlations were found between the plasma level of NT-proBNP and EF both at rest and after exercise $(\mathrm{p}<0.0001)$, whereas positive moderate correlations were found between NT-proBNP and ESV
Table 2. Baseline echocardiographic characteristics - all groups $(n=100)$.

\begin{tabular}{lc}
\hline ECHO rest & $54.0 \pm 6.0$ \\
LVDD [mm] & $40.5 \pm 7.4$ \\
LVDS [mm] & $125.8 \pm 46.9$ \\
LVEDV [mL] & $73.9 \pm 38.6$ \\
LVESV [mL] & $44.0 \pm 9.3$ \\
EF rest [\%] & $1.57 \pm 0.3$ \\
WMSI rest & $23.7 \pm 8.5$ \\
TRPG rest [mmHg] & $15.0 \pm 2.0$ \\
EROA rest [mm²] & $22.5 \pm 5.5$ \\
MRvol rest [mL] & $0.8 \pm 0.2$ \\
CH rest [cm] & $2.0 \pm 0.6$ \\
TA rest [cm $\left.{ }^{2}\right]$ & $15.0 \pm 4.1$ \\
TAPSE [mm] & $0.38[0.3-0.45]$ \\
Sls & \\
ECHO exercise & $44.8 \pm 9.8$ \\
EF exe [\%] & $33.6 \pm 14.7$ \\
TRPG exe [mmHg] & $18.0 \pm 8.0$ \\
EROA exe [mm $\left.{ }^{2}\right]$ & $26.8 \pm 11.7$ \\
MRvol exe [mL] & \\
\hline
\end{tabular}

Data are presented as mean \pm standard deviation. $\mathrm{CH}-$ coaptation height; $E F$ - ejection fraction; EROA - effective regurgitant orifice area; exe - exercise; LVDD - left ventricular end-diastolic dimension; LVDS - left ventricular end-systolic dimension; LVEDV — left ventricular end-diastolic volume; LVESV - left ventricular endsystolic volume; SIs - sphericity index at end-systole; MRvol mitral regurgitation volume; TA - tenting area; TAPSE - tricuspid annular plane systolic excursion; rest - echo examination at rest; TRPG — maximal tricuspid regurgitant peak gradient; WMSI — wall motion score index

$(\mathrm{p}<0.0001)$ and TA $(\mathrm{p}<0.0001)$ both at rest and after exercise. The correlation coefficients were similar for NT-proBNP at rest and NT-proBNP exe. There were also weak, but significant, correlations between NT-proBNP and other resting or post exercise measurements of LV dimensions (LV end-diastolic dimension, LV end-systolic dimension), right ventricle function assessed using tricuspid annular plane systolic excursion, severity of IMR (EROA, MRvol), and probability of pulmonary hypertension assessed using TRPG. The correlation coefficients for the above-mentioned parameters were similar for NT-proBNP at rest and NT-proBNP exe, and no statistically significant correlation existed between NT-proBNP and patient age. Furthermore, significant changes in IMR severity and maximal TRPG during an exercise test were strongly associated with the level of absolute $\triangle$ NT-proBNP after exercise. A significant positive correlation was found between the level of abso- 
Table 3. Correlation between the plasma level of NT-proBNP and clinical and echocardiographic measures at rest and at peak exercise.

\begin{tabular}{lcccccc}
\hline & $\begin{array}{c}\text { NT-proBNP } \\
\text { at rest, r value }\end{array}$ & P value & $\begin{array}{c}\text { NT-proBNP exe, } \\
\text { r value }\end{array}$ & P value & $\begin{array}{c}\Delta \text { BNP, } \\
\text { r value }\end{array}$ & P value \\
\hline Age & 0.11389 & 0.2617 & 0.11966 & 0.2381 & 0.00469 & 0.9633 \\
WAT & -0.29797 & 0.0027 & -0.28685 & 0.0040 & 0.00170 & 0.9867 \\
LVDD & 0.25969 & 0.0094 & 0.25200 & 0.0119 & 0.19841 & 0.0490 \\
LVDS & 0.34539 & 0.0005 & 0.33043 & 0.0008 & 0.19839 & 0.0490 \\
SIs & 0.33863 & 0.0006 & 0.33315 & 0.0008 & 0.19755 & 0.05 \\
LVESV & 0.43340 & $<0.0001$ & 0.42522 & $<0.0001$ & 0.26054 & 0.0092 \\
EF rest & -0.51588 & $<0.0001$ & -0.50414 & $<0.0001$ & -0.28304 & 0.0045 \\
EF exe & -0.57531 & $<0.0001$ & -0.57102 & $<0.0001$ & -0.37037 & 0.0002 \\
TAPSE & -0.21553 & 0.0322 & -0.21390 & 0.0335 & -0.06326 & 0.5339 \\
TRPG rest & 0.2 & 0.0471 & 0.20376 & 0.0431 & 0.21811 & 0.0301 \\
TRPG exe & 0.34616 & 0.0004 & 0.36661 & 0.0002 & 0.44151 & $<0.0001$ \\
EROA rest & 0.27049 & 0.0068 & 0.26598 & 0.0078 & 0.24631 & 0.0140 \\
EROA exe & 0.29030 & 0.0036 & 0.31848 & 0.0013 & 0.49240 & $<0.0001$ \\
MRvol rest & 0.17308 & 0.0867 & 0.17990 & 0.0748 & 0.32621 & 0.001 \\
MRvol exe & 0.31338 & 0.0016 & 0.33844 & 0.0006 & 0.47944 & $<0.0001$ \\
CH rest & 0.3487 & 0.0004 & 0.35787 & 0.0003 & 0.44185 & $<0.0001$ \\
TA rest & 0.43884 & $<0.0001$ & 0.44 & $<0.0001$ & 0.42485 & $<0.0001$ \\
\hline
\end{tabular}

WAT — workload in watts; rest abbreviations — see Tables 1 and 2

Table 4. Multivariate stepwise logistic regression analysis for the prediction of a significant increase in mitral regurgitation severity and pulmonary arterial pressure during exercise.

\begin{tabular}{lcc}
\hline & F value & P value \\
\hline EROA $\geq \mathbf{2 0} \mathbf{~ m m}^{\mathbf{2}}$ & & \\
TA rest & 28.48 & 0.0001 \\
$\Delta$ NT-proBNP & 4.66 & 0.03 \\
TRPG $\geq \mathbf{5 0 ~} \mathbf{m m H g}$ & & \\
CH rest & 12.76 & 0.0006 \\
$\Delta$ NT-proBNP & 4.65 & 0.03 \\
\hline
\end{tabular}

Abbreviations - see Tables 1 and 2

lute $\triangle$ NT-proBNP and EROA, MRvol, and TRPG at peak exercise ( $p<0.001$ for each). There was also a positive moderate correlation between $\triangle \mathrm{NT}$ -proBNP and TA at rest $(\mathrm{p}<0.0001)$. Multivariate stepwise logistic regression analysis showed that the levels of absolute $\Delta \mathrm{NT}$-proBNP and TA at rest were the only independent predictors of the development of severe IMR at peak exercise $(\mathrm{p}=0.033$ and $\mathrm{p}<0.0001$, respectively). Absolute $\triangle \mathrm{NT}$-proBNP and $\mathrm{CH}$ were independent predictors of a significant increase in TRPG at peak exercise $(p=0.033$ and $p=0.0006$, respectively) (Table 4).

The AUC for $\triangle \mathrm{NT}$-proBNP predicting an increased EROA of $\geq 20 \mathrm{~mm}^{2}$ was $0.812(95 \% \mathrm{CI}$ 0.721-0.884; $p=0.0001$; Fig. 1). The absolute increase in NT-proBNP level of $>68.9 \mathrm{pg} / \mathrm{mL}$ was the optimal cutoff, with a sensitivity of $67 \%$ and a specificity of $90 \%$. For NT-proBNP exe, the AUC was 0.702 (95\% CI 0.602-0.790; $\mathrm{p}=0.0003)$, with an optimal cutoff of $924 \mathrm{pg} / \mathrm{mL}$, sensitivity of $67 \%$, and specificity of $74 \%$. For NT-proBNP at rest, the AUC was 0.685 (95\% CI 0.583-0.774; $\mathrm{p}=0.0012$ ); the optimal cutoff was $596 \mathrm{pg} / \mathrm{mL}$, with sensitivity of $83 \%$ and specificity of $55 \%$. The AUC that predicted an increased TRPG of $\geq 50 \mathrm{mmHg}$ at peak exercise for $\triangle$ NT-proBNP was $0.842(95 \%$ CI 0.756-0.908; $p=0.0001$; Fig. 2). The absolute increase of NT-proBNP level of $>68 \mathrm{pg} / \mathrm{mL}$ was the optimal cutoff, with a sensitivity of $80 \%$ and specificity of $76 \%$. For NT-proBNP exe, the AUC was 0.768 (95\% CI $0.672-0.847 ; \mathrm{p}=0.004)$, with an optimal cutoff of $1015 \mathrm{pg} / \mathrm{mL}$, sensitivity of $87 \%$, and specificity of $74 \%$. For NT-proBNP at rest, the AUC was 0.756 (95\% CI 0.659-0.837; $\mathrm{p}=0.0008$ ), with an optimal cutoff of $926 \mathrm{pg} / \mathrm{mL}$, sensitivity of $87 \%$, and specificity of $73 \%$. The 


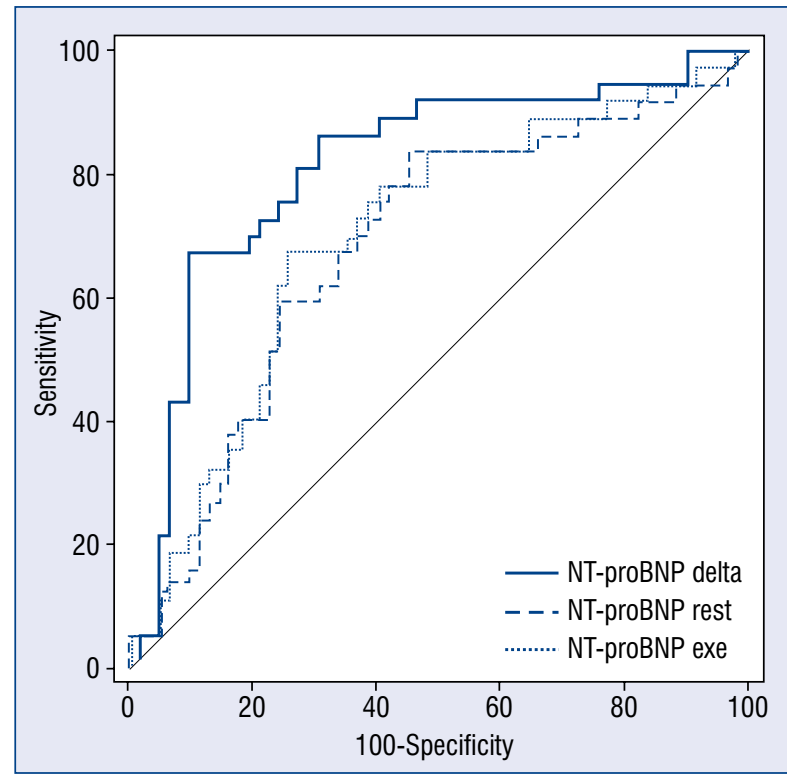

Figure 1. Receiver-operating characteristic curve analysis for $\mathrm{N}$-terminal fragment B-type natriuretic peptide (NT-proBNP) at rest, NT-proBNP exe, and $\triangle \mathrm{NT}$-proBNP as predictors of reaching exercise-induced severe mitral regurgitation (EROA $\geq 20 \mathrm{~mm}^{2}$ ).

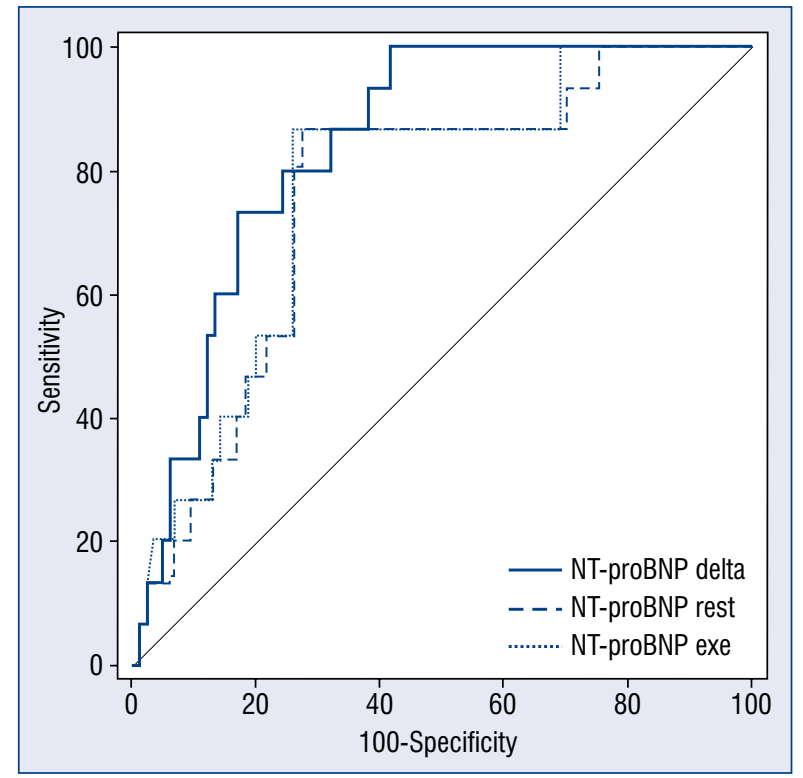

Figure 2. Receiver-operating characteristic curve analysis for $\mathrm{N}$-terminal fragment $\mathrm{B}$-type natriuretic peptide (NT-proBNP) at rest, NT-proBNP exe, and $\triangle \mathrm{NT}$-proBNP as predictors of reaching exercise-induced significant pulmonary hypertension (TRPG $\geq 50 \mathrm{mmHg}$ ).

Table 5. Sensitivity, specificity, and area under the curve (AUC) of NT-proBNP at rest, NT-proBNP exe, and $\triangle \mathrm{NT}$-proBNP for prediction of exercise-induced pulmonary hypertension and severe mitral regurgitation.

\begin{tabular}{lcccc}
\hline & Cutoff $[\mathrm{pg} / \mathrm{mL}]$ & Sensitivity [\%] & Specificity [\%] & AUC \\
\hline EROA $\geq \mathbf{2 0} \mathbf{~ m m}^{\mathbf{2}}$ & & & & \\
NT-proBNP at rest & 596 & 83 & 55 & 0.685 \\
NT-proBNP exe & 924 & 67 & 74 & 0.702 \\
NNT-proBNP & 68.9 & 67 & 90 & 0.812 \\
TRPG $\geq \mathbf{5 0} \mathbf{~ m m H g}$ & & & & \\
NT-proBNP at rest & 926 & 87 & 73 & 0.756 \\
NT-proBNP exe & 1015 & 87 & 74 & 0.768 \\
$\Delta$ NT-proBNP & 68 & 80 & 76 & 0.842 \\
\hline
\end{tabular}

Abbreviations - see Tables 1 and 2

ROC analysis confirmed the high predictive value of $\triangle$ NT-proBNP at peak exercise for reaching the analyzed endpoints (increased EROA of $\geq 20 \mathrm{~mm}^{2}$ and TRPG $\geq 50 \mathrm{mmHg}$ at peak exercise). Table 5 presents the sensitivity, specificity, and AUC of the $\triangle$ NT-proBNP level for predicting abnormal peak-exercise pulmonary pressure thresholds and a significant increase in IMR severity (EROA $\geq 20 \mathrm{~mm}^{2}$ ) at peak exercise.
Analyses of the ROC curves for EROA $\geq 20 \mathrm{~mm}^{2}$ also indicated differences in the AUCs for NT-proBNP at rest, NT-proBNP exe, and $\triangle$ NT-proBNP. Pairwise comparisons of the ROC curves showed significant differences between $\triangle$ NT-proBNP and NT-proBNP at rest ( $\mathrm{p}=0.006)$, as well as between $\triangle$ NT-proBNP and NT-proBNP exe $(p=0.011)$. Similar results were not observed in the analysis of the ROC curves for TRPG $\geq 50 \mathrm{mmHg}$ (Figs. 1, 2). 


\section{Discussion}

Secondary IMR considerably worsens the prognosis of patients after acute MI, both in the short- and long-term follow-up $[1,5]$. It is difficult to elaborate on uniform treatment standards in this group of patients because of the complex mechanism and dynamic nature of IMR. In many patients, significant changes in IMR severity during exercise can be observed independent of the degree of IMR at rest [1-3]. Surgical correction should be considered in patients with severe secondary IMR undergoing CABG, but optimal management of moderate IMR in patients qualified for $\mathrm{CABG}$ remains controversial [17-18, 28, 29].

When the complex and dynamic nature of IMR is taken into consideration, it seems that a more precise qualification of a patient to a proper surgical procedure should be broadened by the evaluation of the IMR variability in the exercise test as well as myocardial viability during DSE. The most important element of the analysis was the assessment of the relationships of the NT-proBNP levels, LV function, IMR severity, and maximal TRPG changes at rest and during exercise, observed through echocardiography.

It was hypothesized that the NT-proBNP level with exercise could be a reliable parameter in predicting a significant increase in IMR severity and pulmonary systolic artery pressure and may help in the identification of high-risk patients with moderate IMR qualified for surgical revascularization. The presumption was that this would help in identifying subgroups of patients who could benefit from better qualification to different surgical strategies (CABG alone or CABG with mitral repair).

In the present study, observations revealed a significant correlation of NT-proBNP levels at rest and at peak exercise with LV volumes and function. Yusoff et al. [11] reported statistically significant associations between the plasma level of N-terminal-BNP and LV end systolic volumes in patients with severe non-ischemic mitral regurgitation. Detaint et al. [30] found LV end-systolic volume index as the major determinant of the plasma level of BNP. Herein, it was shown that in patients with moderate IMR, an absolute increase in the NT-proBNP level at peak exercise was significantly correlated with changes in IMR severity and maximal TRPG at peak exercise. It has been shown that BNP concentrations are strictly associated with hemodynamic status in patients with heart failure [31]. It was known that in some patients with moderate IMR at rest, an increase in mitral regurgitation severity and TRPG is associated with an increase in NT-proBNP levels during exercise. Natriuretic peptides are usually produced by ventricular and atrial myocytes in response to increases in LV stress, and by atrial myocytes in response to increased atrial wall stress [8, 9, 32]. In patients with IMR, the predominant pressure load in mitral regurgitation is on the left atrium; in some patients it is on the right ventricle if there is an increase in pulmonary pressure [10-11]. The strong association between an exercise-induced absolute increase in NT-proBNP levels and pulmonary artery pressure also raises the possibility of increased NT-proBNP secretion from the right ventricle. The present data supports those obtained by Kerr et al. [14], who demonstrated that in patients with moderate to severe or severe MR and preserved resting LV EF, an increase in BNP level is associated with pulmonary artery hypertension on exercise and left atrial enlargement. The results of the current study suggest that in the analyzed models, only $\triangle \mathrm{NT}$-proBNP and TA at rest as well as $\triangle \mathrm{NT}$-proBNP and $\mathrm{CH}$ value at rest were good predictors of an increase in TRPG of $\geq 50 \mathrm{mmHg}$ and in EROA of $\geq 20 \mathrm{~mm}^{2}$ at peak exercise, respectively. An absolute increase in the NT-proBNP level was a more important predictor of the analyzed endpoint than were the levels of NT-proBNP at rest and at peak exercise.

According to available research, this is the first prospective study to investigate the relation between changes in NT-proBNP with exercise and IMR severity, as well as the pulmonary pressure at rest and at peak exercise in patients with moderate IMR qualified for CABG. At this time combined surgery is more likely to be considered if myocardial viability as well as exercise-induced symptoms and increase in IMR severity are present.

Based on the present data, it can be speculated that integration of the assessment of NT-proBNP at rest and at exercise might improve patient selection for valve surgery

\section{Limitations of the study}

A limitation of this study is that it does not include follow-up for clinical outcome or reassessment of NT-proBNP level after surgery.

The study was a part of a grant from the Ministry of Science and Higher Education and not every routine echo parameter was analyzed.

Although the relationships between NTproBNP and both pulmonary pressures and IMR severity are statistically significant, the predictive value of NT-proBNP for individual pa- 
tients requires further study using a larger cohort. Therefore, larger prospective studies should be conducted and the use of NT-proBNP in the diagnostic workup of patients with moderate IMR should be considered.

The present study used EROA and MRvol as reference parameters to estimate the degree of mitral regurgitation. Unfortunately, the regurgitant orifice is often non-spherical shaped in ischemic mitral regurgitation. In such cases, using two-dimensional PISA method may result in underestimation of IMR. Moreover, when IMR is not holosystolic, the degree of IMR may be overestimated [33]. However, EROA and MRvol are the most repeatable parameters of mitral regurgitation and using of three-dimensional echocardiography is very difficult in exercise echocardiography. Moreover, previous studies suggest that absolute measurements of EROA and MRvol provide the strongest predictors of outcome.

\section{Conclusions}

The level of absolute $\Delta$ NT-proBNP during exercise has been found to be the most important parameter in predicting significant changes in IMR severity and pulmonary pressure following exercise. Thus, data herein suggests that NT-proBNP measurement during exercise might be of diagnostic value with therapeutic implications. This hypothesis must be confirmed in larger multicenter studies.

\section{Funding}

The study was supported by a grant from the Ministry of Science and Higher Education (No. 2 P 05B 080 29).

\section{Conflict of interest: None declared}

\section{References}

1. Piérard L, Lancellotti P. The role of ischemic mitral regurgitation in the pathogenesis of acute pulmonary edema. $\mathrm{N}$ Engl J Med. 2004; 351(16): 1627-1634, doi: 10.1056/nejmoa040532, indexed in Pubmed: 15483281.

2. Lancellotti P, Troisfontaines P, Toussaint AC, et al. Prognostic importance of exercise-induced changes in mitral regurgitation in patients with chronic ischemic left ventricular dysfunction. Circulation. 2003; 108(14): 1713-1717, doi: 10.1161/01. cir.0000087599.49332.05.

3. Lancellotti P, Piérard LA. Chronic ischaemic mitral regurgitation: exercise testing reveals its dynamic component. Eur Heart J. 2005; 26: 1816-1817.

4. Lancellotti P, Lebrun F, Pierard LA. Determinants of Exerciseinduced changes in mitral regurgitation in patients with coronary artery disease and left ventricular dysfunction. ACC Curr J Rev. 2004; 13(4): 28, doi: 10.1016/j.accreview.2004.03.009.

5. Grigioni F, Enriquez-Sarano M, Zehr KJ, et al. Ischemic mitral regurgitation. Long-term outcome and prognostic implications with quantitative Doppler assessment. ACC Curr J Rev. 2001; 10(5): 33, doi: 10.1016/s1062-1458(01)00387-7.

6. Lancellotti P, Stainier PY, Lebois F, et al. Effect of dynamic left ventricular dyssynchrony on dynamic mitral regurgitation in patients with heart failure due to coronary artery disease. Am J Coll. 2005; 96(9): 1304-1307, doi: 10.1016/j.amjcard.2005. 06.077, indexed in Pubmed: 16253603.

7. Sztefko K. NT-proBNP: a biomarker with new potential application. Pol Arch Med Wewn. 2015; 125(7-8): 509-510, doi: 10.20452/pamw.2989.

8. Levin E, Gardner D, Samson W, et al. Natriuretic peptides. N Engl J Med. 1998; 339(5): 321-328, doi: 10.1056/ nejm199807303390507, indexed in Pubmed: 9682046.

9. Cheung B, Kumara CR. Natriuretic peptides - relevance in cardiovascular disease. JAMA. 1998; 280(23): 1983, doi: 10.1001/ jama.280.23.1983, indexed in Pubmed: 9863839.

10. Sutton T, Stewart R, Gerber I, et al. Plasma natriuretic peptide levels increase with symptoms and severity of mitral regurgitation. J Am Coll Cardiol. 2003; 41(12): 2280-2287, doi: 10.1016/ s0735-1097(03)00486-8.

11. Yusoff R, Clayton N, Keevil B, et al. Utility of plasma N-terminal brain natriuretic peptide as a marker of functional capacity in patients with chronic severe mitral regurgitation. Am J Cardiol. 2006; 97(10): 1498-1501, doi: 10.1016/j.amjcard.2005.11.085, indexed in Pubmed: 16679092.

12. Foote RS, Pearlman JD, Siegel AH, et al. Detection of exerciseinduced ischemia by changes in B-type natriuretic peptides. ACC Curr J Rev. 2005; 14(3): 26, doi: 10.1016/j.accreview.2005.02.041.

13. Koç M, Bozkurt A, Acartürk E, et al. Usefulness of N-terminal pro-B-type natriuretic peptide increase with exercise for predicting cardiovascular mortality in patients with heart failure. Am J Cardiol. 2008; 101(8): 1157-1162, doi: 10.1016/j.amjcard.2007.11.070, indexed in Pubmed: 18394451.

14. Kerr AJ, Raffel OC, Whalley GA, et al. Elevated B-type natriuretic peptide despite normal left ventricular function on rest and exercise stress echocardiography in mitral regurgitation. Eur Heart J. 2008; 29(3): 363-370, doi: 10.1093/eurheartj/ehm553, indexed in Pubmed: 18202251.

15. Kilickesmez K, Özkan A, Abaci O, et al. Serum N-terminal brain natriuretic peptide indicates exercise induced augmentation of pulmonary artery pressure in patients with mitral stenosis. Echocardiography. 2010; 28(1): 8-14, doi: 10.1111/j.15408175.2010.01273.x, indexed in Pubmed: 20738368.

16. Dobrowolski P, Lech A, Klisiewicz A, et al. Evaluation of NTproBNP concentrations during exercise in asymptomatic patients with severe high-gradient aortic stenosis. Pol Arch Med Wewn. 2016; 126(9): 635-641.

17. Nishimura RA, Otto CM, Bonow RO, et al. 2017 AHA/ACC Focused Update of the 2014 AHA/ACC Guideline for the Management of Patients With Valvular Heart Disease: A Report of the American College of Cardiology/American Heart Association Task Force on Clinical Practice Guidelines. J Am Coll Cardiol. 2017; 70: 252-289.

18. Baumgartner H, Falk V, Bax JJ, et al. 2017 ESC/EACTS Guidelines for the management of valvular heart disease. Eur Heart J. 2017; 38: 2739-91. 
19. Pierard LA, Carabello BA. Ischaemic mitral regurgitation: pathophysiology, outcomes and the conundrum of treatment. Eur Heart J. 2010; 31(24): 2996-3005, doi: 10.1093/eurheartj/ ehq411, indexed in Pubmed: 21123277.

20. Lancellotti P, Marwick T, Pierard LA. How to manage ischaemic mitral regurgitation. Heart. 2008; 94(11): 1497-1502, doi: 10.1136/hrt.2007.134833, indexed in Pubmed: 18931162.

21. Cerqueira M, Weissman N, Dilsizian V, et al. Standardized myocardial segmentation and nomenclature for tomographic imaging of the heart. Circulation. 2002; 105(4): 539-542, doi: 10.1161/ hc0402.102975, indexed in Pubmed: 11815441.

22. Lang R, Bierig M, Devereux R, et al. Recommendations for Chamber Quantification: A Report from the American Society of Echocardiography's Guidelines and Standards Committee and the Chamber Quantification Writing Group, Developed in Conjunction with the European Association of Echocardiography, a Branch of the European Society of Cardiology. J Am Soc Echocardiogr. 2005; 18(12): 1440-1463, doi: 10.1016/j.echo.2005.10.005.

23. Galiè N, Humbert M, Vachiery JL, et al. 2015 ESC/ERS Guidelines for the diagnosis and treatment of pulmonary hypertension: The Joint Task Force for the Diagnosis and Treatment of Pulmonary Hypertension of the European Society of Cardiology (ESC) and the European Respiratory Society (ERS): Endorsed by: Association for European Paediatric and Congenital Cardiology (AEPC), International Society for Heart and Lung Transplantation (ISHLT). Eur Heart J. 2016; 37(1): 67-119.

24. Yiu S, Enriquez-Sarano M, Tribouilloy C, et al. Determinants of the degree of functional mitral regurgitation in patients with systolic left ventricular dysfunction. Circulation. 2000; 102(12): 1400-1406, doi: 10.1161/01.cir.102.12.1400.

25. Bax J, Poldermans D, Elhendy A, et al. Improvement of left ventricular ejection fraction, heart failure symptoms and prognosis after revascularization in patients with chronic coronary artery disease and viable myocardium detected by dobutamine stress echocardiography. J Am Coll Cardiol. 1999; 34(1): 163-169, doi: 10.1016/s0735-1097(99)00157-6.
26. Pellikka P, Nagueh S, Elhendy A, et al. American Society of Echocardiography Recommendations for Performance, Interpretation, and Application of Stress Echocardiography. J Am Soc Echocardiogr. 2007; 20(9): 1021-1041, doi: 10.1016/j.echo.2007.07.003, indexed in Pubmed: 17765820.

27. DeLong E, DeLong D, Clarke-Pearson D. Comparing the areas under two or more correlated receiver operating characteristic curves: a nonparametric approach. Biometrics. 1988; 44(3): 837, doi: $10.2307 / 2531595$.

28. Kang DH. Mitral valve repair versus revascularization alone in the treatment of ischemic mitral regurgitation. Circulation. 2006; 114(1_suppl): I-499-I-503, doi: 10.1161/circulationaha.105.000398.

29. Fattouch K, Guccione F, Sampognaro R, et al. POINT: Efficacy of adding mitral valve restrictive annuloplasty to coronary artery bypass grafting in patients with moderate ischemic mitral valve regurgitation: A randomized trial. J Thorac Cardiovasc Surg. 2009; 138(2): 278-285, doi: 10.1016/j.jtcvs.2008.11.010, indexed in Pubmed: 19619766.

30. Detaint D, Messika-Zeitoun D, Chen H, et al. Association of B-type natriuretic peptide activation to left ventricular endsystolic remodeling in organic and functional mitral regurgitation. Am J Cardiol. 2006; 97(7): 1029-1034, doi: 10.1016/j. amjcard.2005.10.061, indexed in Pubmed: 16563910.

31. Mueller C, Scholer A, Laule-Kilian K, et al. Use of B-type natriuretic peptide in the evaluation and management of acute dyspnea. N Engl J Med. 2004; 350(7): 647-654, doi: 10.1056/ nejmoa031681, indexed in Pubmed: 14960741.

32. Goetze JP, Friis-Hansen L, Rehfeld JF. Atrial secretion of B-type natriuretic peptide. Eur Heart J. 2006; 27(14): 1648-1650, doi: 10.1093/eurheartj/ehl109, indexed in Pubmed: 16785247.

33. Zoghbi W, Adams D, Bonow R, et al. Recommendations for noninvasive evaluation of native valvular regurgitation: A report from the American Society of Echocardiography developed in collaboration with the society for cardiovascular magnetic resonance. J Indian Acad Echocardiogr Cardiovasc Imag. 2020; 4(1): 58, doi: 10.4103/2543-1463.282191. 